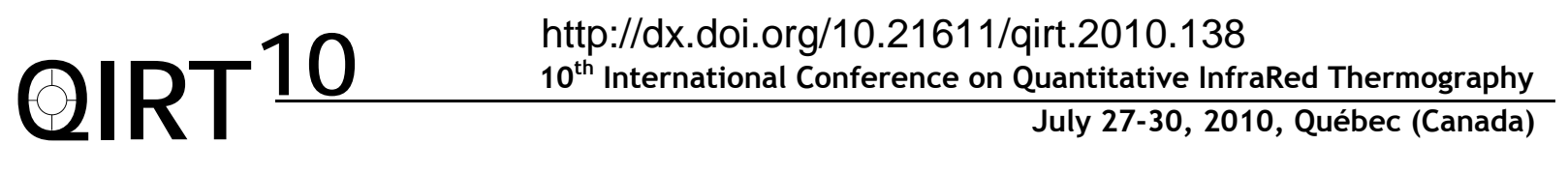

\title{
Estimating and quantifying theoretical errors of temperature measurement in bi- spectral thermography
}

by V. TORTEL, Y. SFAXI and R. KHELALFA

IUT de SENART, CERTES, Université Paris Est Créteil (France), tortel@univ-paris12.fr

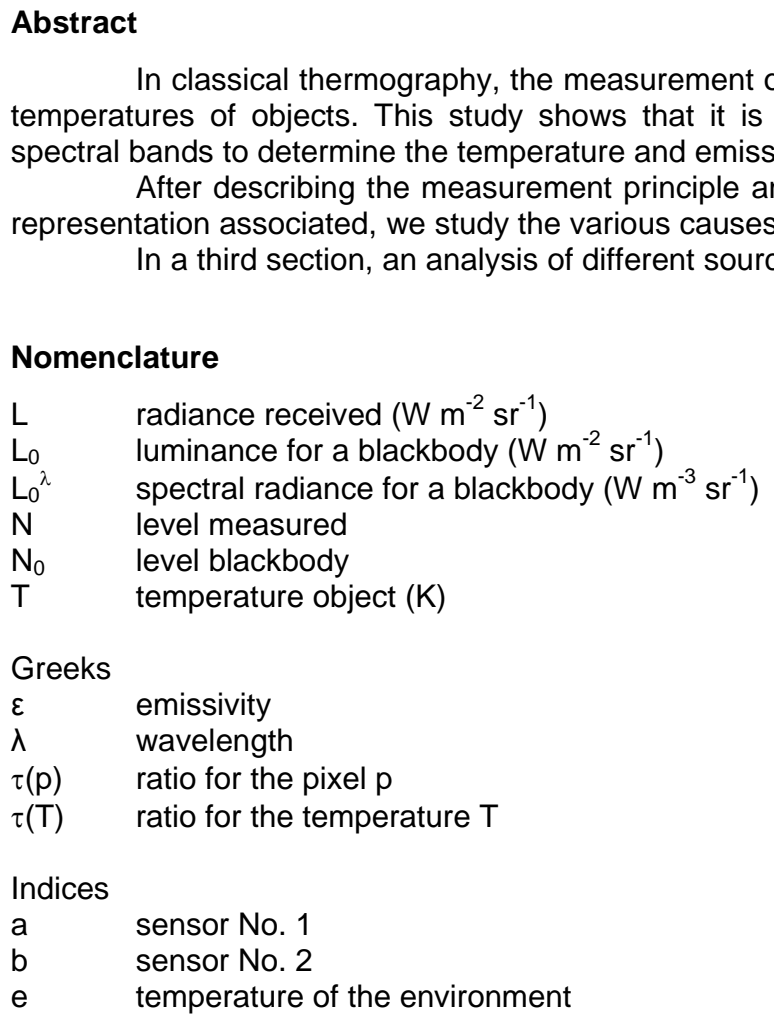

In classical thermography, the measurement of luminance and knowledge of the emissivity can be inferred from the temperatures of objects. This study shows that it is theoretically possible, by measuring the luminance in two different spectral bands to determine the temperature and emissivity of an object.

After describing the measurement principle and the establishment of phenomenological equations and a graphical representation associated, we study the various causes of inaccurate results.

In a third section, an analysis of different sources of errors in experimental measurements will be presented.

\section{Introduction}

A material object whose temperature is not absolute zero emits and absorbs radiation called thermal radiation. The description of the thermal radiation found by Max Planck showed that the radiation is mainly limited to a theoretical maximum depending on the wavelength and temperature. The spectral radiance $\left(\mathrm{W} \mathrm{m}^{-3} \mathrm{sr}^{-1}\right)$ is described by the corresponding Planck law also named luminance of the blackbody.

The Planck law gives the intensity radiated by a blackbody as a function of wavelength (or frequency). Let a blackbody have temperature T. The Planck law can be expressed through the following equation:

$$
L_{0}\left(\lambda_{0} T\right)=\frac{2 h c^{2}}{\lambda^{5}} \frac{1}{e^{\frac{\operatorname{dh} c}{\ln T}-1}}
$$

where $\mathrm{h}$ is Planck's constant, $\mathrm{c}$ is the speed of light, and $\mathrm{k}$ is Boltzmann's constant

The integration of Planck's law over all wavelengths gives Stephan-Boltzmann law describing the total energy flux $\Phi$ emitted from a blackbody at temperature T. It can be computed as: 


$$
\Phi=\sigma T^{4}
$$

where $\sigma$ is a constant called the Stefan-Boltzmann constant.

Real objects emit only a part of the luminance of the blackbody; emissivity indicates the importance of real influence over the blackbody.

In practice, the emissivities are often close to $1(0.8$ to 0.98$)$ except for metals, especially if they are polished, for which emissivities range from 0.02 to 0.1 .

In classical thermography, we consider an object at temperature $\mathrm{T}$ placed in an environment (or background) composed of objects with uniform temperature $T_{\mathrm{e}}$. This background radiation implies an isotropic uniform radiation: the radiation environment.

In these conditions, the spectral brightness of the object is written:

$$
L(\lambda)=\varepsilon \mathbb{L}_{0}^{2}\left(\lambda_{v} T\right)+(1-\varepsilon) L_{0}^{2}\left(\lambda_{v} T_{\varepsilon}\right)
$$

In this relationship, the second term indicates that part of the radiation environment is reflected by the object.

Radiation sensors respond to a range of wavelengths. We must therefore integrate Eq. (3) to determine the measured luminance. This integration gives the radiometric equation:

$$
\mathbb{L}=a L_{0}(T)+(1-\varepsilon) L_{0}\left(T_{\theta}\right)
$$

The function $L_{0}(T)$ is obtained from measurements on a well-controlled laboratory blackbody, this function is also called calibration curve.

The estimate of the emissivity $\varepsilon$ is very delicate. The transition from (4) to $L_{0}(T)$ is an approximation.

But if one avoids the areas corresponding to metals, the emissivities remain within a limited range, the strong dependency of $L_{0}(T)$ with respect to $T$, limits errors on $T$ due to incorrect estimation of $\varepsilon$.

\section{Principle of the bi-spectral thermography}

In bi-spectral thermography, radiation is received by two sensors in different spectral bands. The responses of both sensors allow to determine the temperature and emissivity [1] [2]. In this study, we assume, based on the assumption of gray body, the emissivity is constant along the wavelength [3] [4].

For an object emissivity $\varepsilon$ at temperature $\mathrm{T}$, in a radiation environment temperature $\mathrm{Te}$, the responses in arbitrary units (quantization levels, noted a.u.) for two sensors of different spectral bands are:

$$
\begin{aligned}
& N_{a}=\varepsilon N_{o a}(T)+(1-\varepsilon) N_{a d}\left(T_{a}\right) \\
& N_{b}=\varepsilon N_{a b}(T)+(1-\varepsilon) N_{a b}\left(T_{a}\right)
\end{aligned}
$$

The functions $N_{o a}(T)$ and $N_{o b}(T)$ are the calibration curves; they reflect the integration of the Planck curve in different bands. Knowledge of $T_{e}$ as well as measures of brightness in both bands $N_{a}$ and $N_{b}$ allow to find $T$ and $\varepsilon$ by solving the system composed of Eqs. (5) and (6).

\section{Temperatures determination}

Based on the responses of sensors $N_{a}$ and $N_{b}$, we define a ratio in which the values $N_{o b}\left(T_{e}\right)$ and $N_{o a}\left(T_{e}\right)$ are constant for all points of a thermogram and are calculated through calibration curves:

$$
\tau=\frac{N_{b}-N_{a b}\left(T_{Q}\right)}{N_{Q}-N_{a \alpha}\left(T_{Q}\right)}
$$

Eqs. (5) and (6) then allow us to write: 


$$
\tau=\frac{N_{o b}(T)-N_{o b}\left(T_{\theta}\right)}{N_{o d}(T)-N_{o d}\left(T_{\theta}\right)}
$$

This shows that $\tau$ depends only on the temperature $\mathrm{T}$ and not the emissivity. The process to obtain the thermogram is as follows:

From Eq. (8), with measured levels on a blackbody, we calculate $\tau(T)$ for any temperature: $\tau(T)$ is the calibration curve for bi-spectral method. This curve depends on $T_{e}$, but the two individual calibration curves $N_{o a}(T)$ and $N_{o b}(T)$ of the two sensors are sufficient to calculate it.

With Eq. (7) and from the measured $N_{a}$ and $N_{b}$ for each pixel, we calculate the ratio $\tau$. By comparison with the calibration curve, we deduce a temperature for that pixel.

\section{Graphing}

The equations presented above do not allow easy evaluation of different sources of errors and their quantification. A graphical representation gives an intuitive vision of defects and qualities expected in theoretical studies and will also analyze the causes of errors in the experimental cases.

To do this representation we plot a point $(P)$ in a plane whose coordinates are $x=N_{a}$ and $y=N_{b}$ we call it "representative point". We can trace all possible locations for theses points when $T$ is varied without changing $\varepsilon$ or when $\varepsilon$ is varied without changing $T$, this gives a graphical resolution of the system of Eqs. (5) - (6).

It is also useful to plot different points $(P)$ for different pixels of the future thermogram, and to analyze it in terms of $T$ and $\varepsilon$.

\subsection{Isotherms line segments}

For a single temperature $T$ of an object, we study the variation of $N_{b}$ as a function of $N_{a}$ for different values of the emissivity $\varepsilon$. $N_{a}$ varies linearly with $\varepsilon$ between $N_{o a}\left(T_{e}\right)$ for $\varepsilon=0$, and $N_{o a}(T)$ for $\varepsilon=1$. $N_{b}$ varies similarly between $N_{o b}\left(T_{e}\right)$ and $\mathrm{N}_{\mathrm{ob}}(\mathrm{T})$. The representative point then describes a line segment.

Writing $x=N_{a}$ and $y=N_{b}$, the system (5) - (6) allow to check that:

$$
y=\pi(T) \cdot x+\beta
$$

Writing:

$X=N_{o a}(T), Y=N_{o b}(T), X_{e}=N_{o a}\left(T_{e}\right)$ et $Y_{e}=N_{o b}\left(T_{e}\right)$, we obtain :

$$
\tau(T)=\frac{\Psi-\Psi_{S}}{Z-X_{S}}
$$

and:

$$
\beta=\frac{X E_{Q}-Z_{Q}}{Z-X_{Q}}
$$

To set a temperature T, $\beta$ and $\tau$ are constant and Eq. (9) is that of a segment we call "feature segment" for the temperature $\mathrm{T}$.

The two points $P 1$ and $P 2$, the ends of this segment have respective coordinates $\left(X_{e}=N_{a o}\left(T_{e}\right), Y_{e}=N_{b o}\left(T_{e}\right)\right)$ et $\left(X=N_{a o}(T), Y=N_{b o}(T)\right)$ whose values are known by calibration curves of both sensors.

\section{2. blackbody curve}

The endpoints of the segments of coordinates $\left(\mathrm{N}_{\mathrm{ao}}(\mathrm{T}), \mathrm{N}_{\mathrm{bo}}(\mathrm{T})\right)$ are all on a curve with temperature as parameter that we call the blackbody curve. This curve corresponds to the measure made on a blackbody, it is accessible by experiment or predicted by theoretical calculation. The isotherms line segments are chords of this curve. For the segments to be distinct, it is necessary that the curve has a sufficiently strong curvature and of constant sign. 


\section{3. iso-emissivity curve} written:

To determine this curve, we vary the value of T but we keep a single emissivity $\varepsilon$. The system (5) - (6) can be

$$
\begin{aligned}
& x-X_{e}=\varepsilon\left(X-X_{e}\right) \\
& y-Y_{e}=\varepsilon\left(Y-Y_{e}\right)
\end{aligned}
$$

with :

$X=N_{o a}(T)$ et $Y=N_{o b}(T)$

$X_{e}=N_{o a}\left(T_{e}\right)$ et $Y_{e}=N_{o b}\left(T_{e}\right)$

Each point of coordinates $(x, y)$ is the result by an homothety of ratio $\varepsilon$ and center $\left(X_{e}, Y_{e}\right)$ of a point of blackbody curve $(X, Y)$. If we consider the whole range of temperatures, we obtain the a new curve with same shape as the blackbody curve.

\subsection{Affine transformation}

In practice the curve of blackbody has a low curvature; the chords of this curve (the isotherms line segments) are not sufficiently distinct. To make the plot more readable we replace y by:

$$
y^{\oplus}=y-\mathbb{k} x
$$

We choose the constant $k$ equal to the average slope of the curve of blackbody. Such a transformation preserves the alignments (ie segments) and homotety, and allows showing clearly the residual curvature (figure 1).

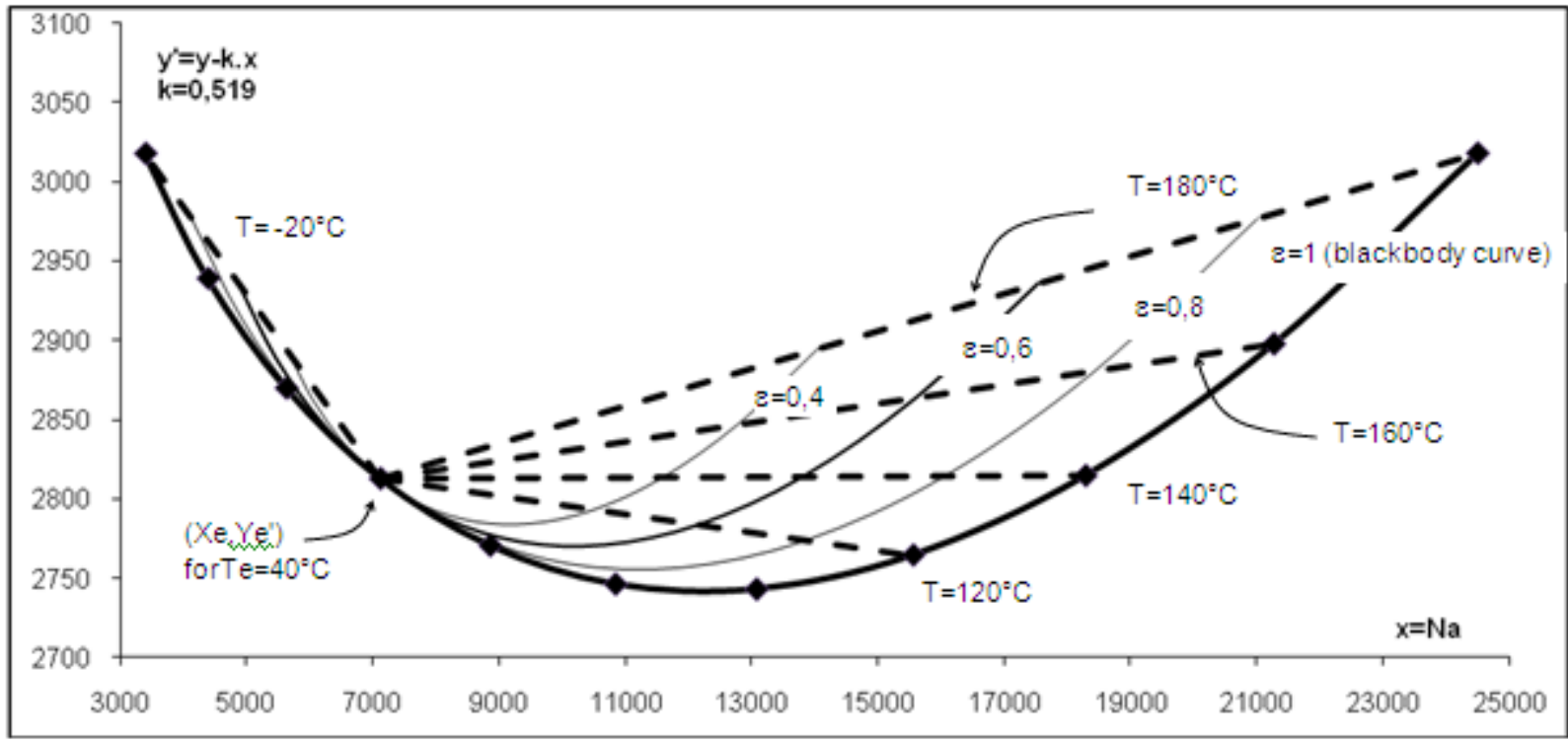

Fig. 1: plot obtained from a blackbody curve calculated (bands 8 microns and 8 microns $10 u . m-12-\mu m$ temperatures $-20^{\circ} \mathrm{C}$ to $\left.180^{\circ} \mathrm{C}\right)$.

The planned locations of representative points for isotherms objects (dashed) and for objects of constant emissivity (solid line) are shown. 


\section{Different sources of errors} errors are:

Some errors can distort the graph or affect the numerical solution by giving wrong values for $\tau(T)$ and for $T$. These

\subsection{Noise in the responses of sensors}

This noise is shown by error bars on each "representative point", because of the affine transformation the rectangular area error is transformed into a parallelogram. With scales used, only the overall height of this parallelogram is visible.

\subsection{Non-gray body}

If there are two different values of emissivity $\varepsilon_{a}$ and $\varepsilon_{b}$ in Eqs. (5) - (6) then "representative point" are no more on the isotherm line segment (figure 2).

If, for example, $\varepsilon_{b}=1,01 \varepsilon_{a}$, then Eqs. (5) - (6) represent a modified line segment whose ends are $P 1\left(X_{e}, Y_{e}\right)$ and $P 2{ }^{\prime}\left(X, Y+0,01 .\left(Y-Y_{e}\right)\right)$. Estimates $Y-Y_{e} \approx k$. $\left(X-X_{e}\right)$ gives a gap $\left\{0,01 k\right.$. $\left.\left(X-X_{e}\right)\right\}$ between points $P 2$ ' and $P 2$. Applied to a temperature $\mathrm{T}=160^{\circ} \mathrm{C}$, with the same parameters as in figure 1 , this gap is 74 a.u. and the effect is very strong as it corresponds to an apparent temperature $\mathrm{T}^{\prime}$ of about $177^{\circ} \mathrm{C}$.

It is also possible to estimate $\left(\varepsilon_{\mathrm{b}} / \varepsilon_{\mathrm{a}}\right)$ in the experimental cases where we know $\mathrm{T}$ : first trace, the segment passing through the point representing measured, then note how far it is above the point P2 of a gray body and then divide dividing by $\mathrm{Y}-\mathrm{Y}_{\mathrm{e}}$.

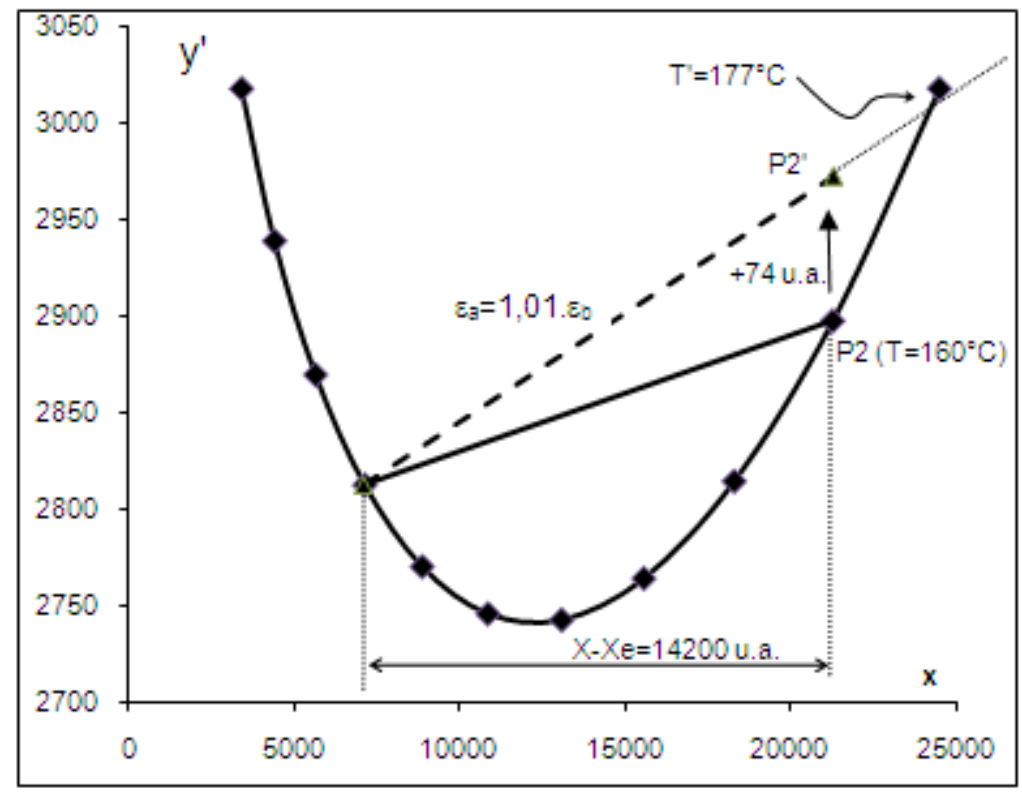

Fig. 2: Effect of a non-gray body: solid line segment for a gray body, dashed line for a non-gray body.

\subsection{Variation of temperature of the object or the environment between the two measures}

Both sets of measurements must be made quickly so that the temperatures of the object or the environment do not change between the two measures.

If, for example, we note the value of $\mathrm{N}_{a}$ at a temperature $\mathrm{T}=160^{\circ} \mathrm{C}$ and we note the value of $\mathrm{N}_{\mathrm{b}}$ at a temperature $\mathrm{T}$ $=161^{\circ} \mathrm{C}$, the representative point $\mathrm{P} 2^{\prime}$ is located 90 a.u. above the normal point, this point is on segment $\mathrm{T}=182^{\circ} \mathrm{C}$ : the variation of $1^{\circ} \mathrm{C}$ leads to an error of $22^{\circ} \mathrm{C}$ for $\mathrm{T}^{\prime}$ (figure 3 ). 


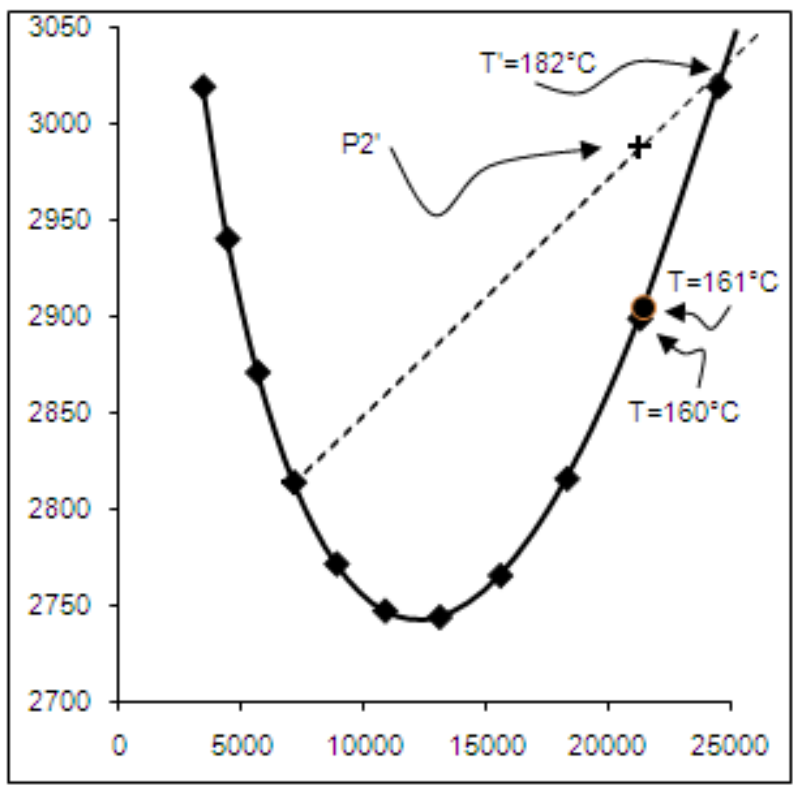

Fig. 3: Effect of a variation of $T$ between measurements of $N_{a}, N_{b}$

\subsection{Sensor Drift}

If the answer of one of the sensors drifts, then all representative points are shifted, the graph is translated. It is possible to compensate for this effect if there is an object of known temperature and emissivity or a blackbody constant temperature $T_{\text {ref }}$ in the field of thermogram. This temperature must be precisely known or constant. With the parameters of figure 1 , a $1^{\circ} \mathrm{C}$ error for $\mathrm{T}_{\text {ref }}$ (around $40^{\circ} \mathrm{C}$ ) gives an error not compensated on $\mathrm{N}_{\mathrm{b}}$ around 40 a.u., the graph remains strongly shifted.

In our experiment, the filter can absorb radiation, so it is also emissive. An estimate shows that an $1^{\circ} \mathrm{C}$ increase of temperature of the filter causes a drift of all the points representing 40 levels up.

\subsection{Space lags between the two measures}

When using for two sensors one single camera with filter and without filter, the offsets are small (around the pixel). The only points that causes problem are those where the contrast is strong: theses are the boundaries of objects and boundaries of emissivity areas. For these points measures $\mathrm{N}_{a}$ and $\mathrm{N}_{b}$ are not connected, the temperature calculated with these measuring points is meaningless.

\section{Experimental Results}

We analyze different sources of errors for the results of two measurement series.

In the first series, we have used a camera "Inframetrics PM295 (8 $\mu \mathrm{m}-12 \mu \mathrm{m}$ band)": it is the sensor a. We then used the same camera with a filter made of $3 \mathrm{~mm}$ thick Fluorite in front of the lens, the filter absorbs wavelengths greater than 10 microns, so we get a measure from $8 \mu \mathrm{m}$ to $10 \mu \mathrm{m}$. We identify "the camera + the filter" to the sensor $\mathrm{b}$. After a series of measures to determine the blackbody calibration curves (two thermograms for each point on the blackbody curve), we observed the responses of the sensors $a$ and $b$ for a single temperature $T=180^{\circ} \mathrm{C}$ and for four different emissivities. This observation was made on an isothermal block painted with 4 different areas (two thermograms for all points $\mathrm{T}=180^{\circ} \mathrm{C}$ ) (figure 4). 


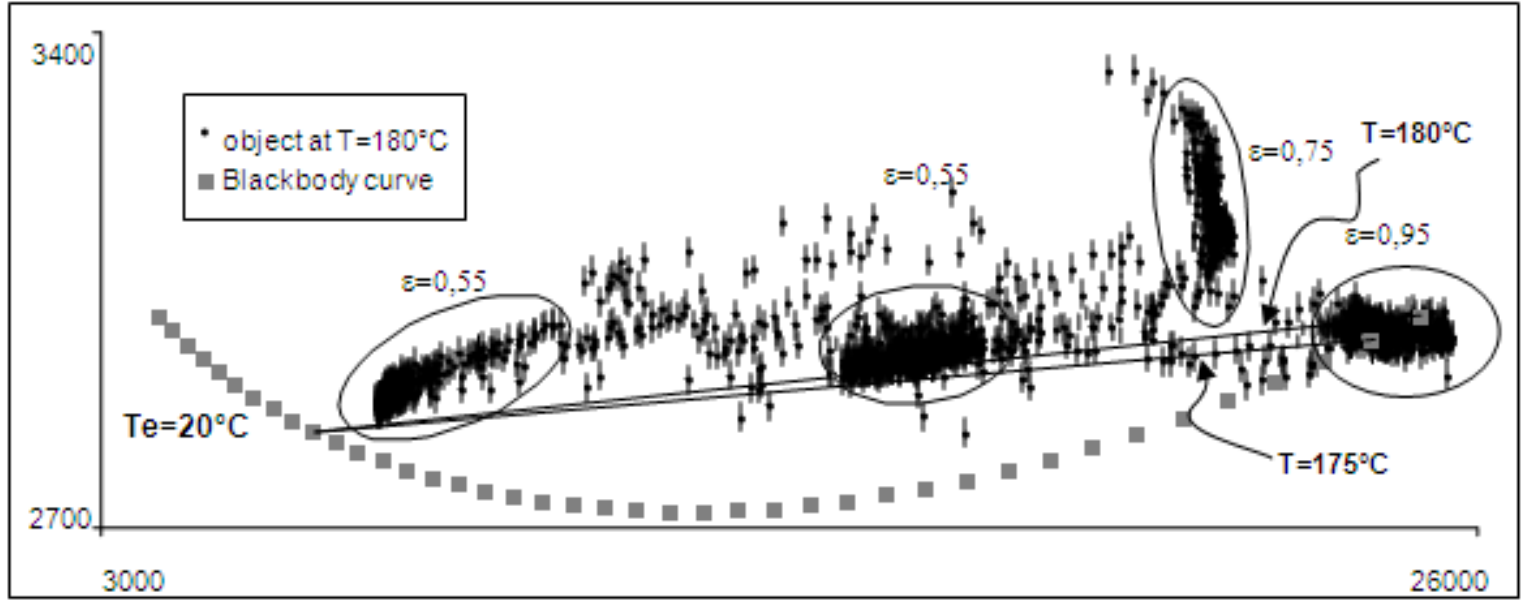

Fig. 4: First measurement series

In the second series of measurements we used a camera "ThermaCAM E300" (7.5 $\mu \mathrm{m}-13 \mu \mathrm{m}$ band), with the same fluorite filter. We recorded the responses of sensors $a$ and $b$ for different temperatures and emissivities successively. Each point on the diagram corresponds to two thermograms in which we take some average values.

The different emissivities are simulated by a blackbody in front of which we put an optical chopper with opening rates $60 \%, 70 \%, 80 \%$ and $90 \%$. For $\varepsilon=1$, we remove the chopper. This method allows us to avoid the problem of gray body and it gives a known emissivity (figure 5).

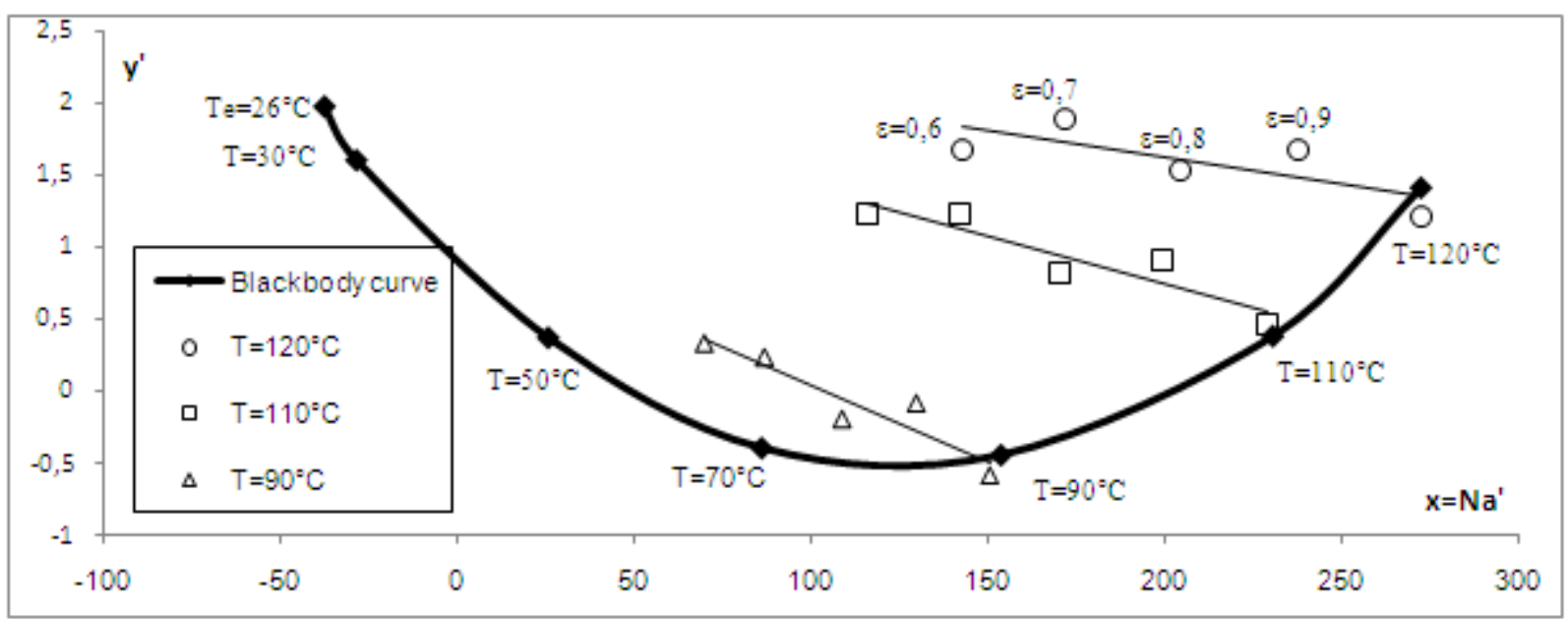

Fig. 5: Second measurement series

The values of $x$ and $y^{\prime}$ are modified to compensate for drift (which explains the negative values), the scale in a.u. is not the same as in figure 4 since it is a different camera.

\section{Analysis of various sources of errors of experimental measurements}

\subsection{Noise of measurement}

Error bars representing the measurement noise are shown in figure 4 . The error bar for a point touch both the $175^{\circ}$ $\mathrm{C}$ and $180^{\circ} \mathrm{C}$ line segments: the error on the apparent temperature is $5^{\circ} \mathrm{C}$ and, for $\varepsilon=0.5$, the error is multiplied by two. 


\subsection{Non-gray body}

In figure 4 , the area of very low emissivity $(\varepsilon \approx 0,05)$ behaves as if $\varepsilon_{a}=1,05 \varepsilon_{b}$. The distance between points and the theoretical segment for the average emissivity zone allow to estimate $\varepsilon_{\mathrm{a}}=0.75$ and $\varepsilon_{\mathrm{b}}=0.76$ to 0.78 .

Slight differences between emissivity cause great effects on the apparent temperature T'.

\subsection{Drift of sensors}

For figure 5 we used an object reference $\left(N_{\text {aref }}, N_{\text {bref, }}\right)$ at a stabilized temperature to detect and correct drift. This correction consists in taking $\mathrm{N}_{a}$ and $\mathrm{N}_{b^{\prime}}$ in place of $\mathrm{N}_{\mathrm{a}}$ and $\mathrm{N}_{\mathrm{b}}$ as follow:

\section{$\mathrm{N}_{\mathrm{a}}{ }^{\prime}=\mathrm{N}_{\mathrm{a}}-\mathrm{N}_{\text {aref }}$}

$\mathrm{N}_{\mathrm{b}}{ }^{\prime}=\mathrm{N}_{\mathrm{b}}-\mathrm{N}_{\text {bref. }}$

The following figures show the curves obtained, without drift compensation (Fig. 6a) and with compensation (Fig. $6 \mathrm{~b})$. The points of figure $6 \mathrm{a}$ have two times greater dispersal than those of figure $6 \mathrm{~b}$. In addition, the curve for the blackbody of figure $6 a$ is shifted.

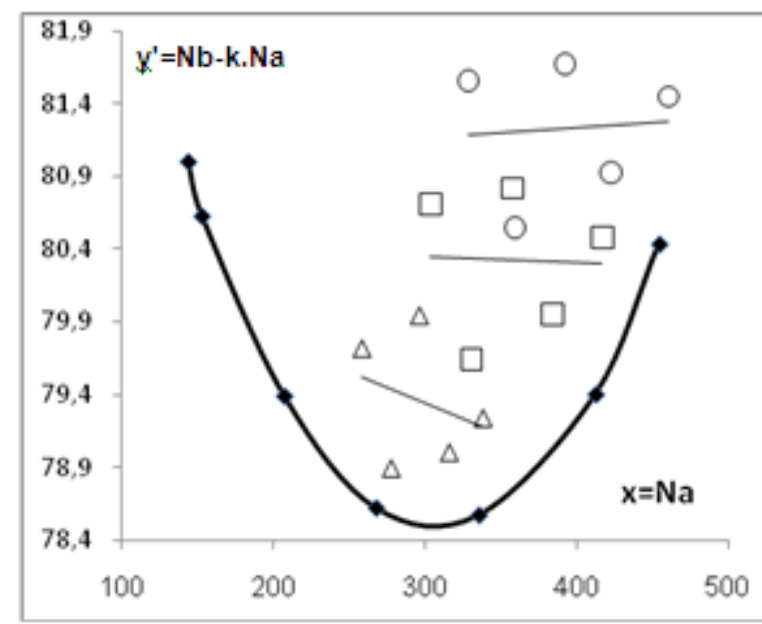

Fig. $6 a$

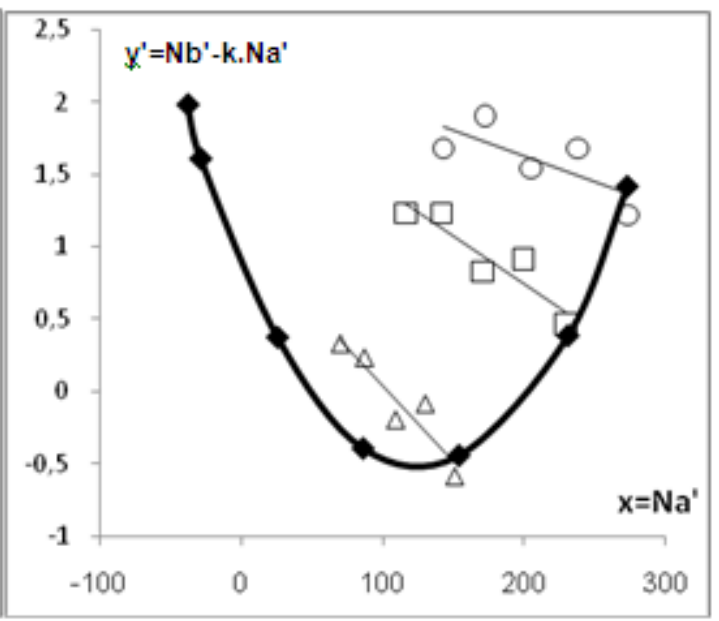

Fig. $6 b$

Fig. 6: Improving with drift compensation

The correction significantly improves the alignments but we had an insufficient stabilization of the reference object (variations of $0.5^{\circ} \mathrm{C}$ ). A remaining drift is easily identifiable because it affects similarly measures done at close times for isotherm segments and for the back of image (not shown).

\section{Conclusion}

The proposed plot is easy to implement and allows a detailed assessment of results. It also allows for performance prediction.

\section{REFERENCES}

[1] [1] K. Chrzanowski, Remote température measurement of highly reflecting objects in outdoor conditions, QIRT 1992 p.32-35.

[2] [2] A.-C. Legrand, P. Gorria, F. Meriaudeau, Caractérisation d'un système multispectral de mesure de température, C2I 2001 p.291-297. Edition Hermès science publications.

[3] [3] V. Tortel, J.-F. Durastanti, D. Glao, J.-P. Bellanger, Mesurage de température par thermographielR à l'aide de filtres, Congrès SFT 2000 p.637-642. Editions Elsevier.

[4] [4] V. Tortel, La thermographie bispectrale, Thermogram' 2001 p.219-246. 